\title{
Generation of dissipative soliton resonance in a fiber laser based on a nonlinear optical loop mirror
}

\author{
M. Durán-Sánchez ${ }^{a}$, R. I. Álvarez-Tamayo ${ }^{b}$, B. Posada-Ramírez ${ }^{c}$, B. Ibarra-Escamilla ${ }^{c}$, \\ M. Bello-Jimenez ${ }^{d}$, I. Armas-Rivera ${ }^{c}$, P. Prieto-Cortés ${ }^{d}$ and E. A. Kuzin ${ }^{c}$ \\ ${ }^{a}$ Conacyt-Instituto Nacional de Astrofísica, Óptica y Electrónica, \\ L. E. Erro 1, Puebla 72824, México; \\ e-mail: mduransa@conacyt.mx \\ ${ }^{b}$ Universidad Popular Autónoma del Estado de Puebla, Puebla 72410, México; \\ e-mail: ricardoivan.alvarez01@upaep.mx \\ ${ }^{c}$ Instituto Nacional de Astrofísica, Óptica y Electrónica, L. E. Erro 1, Puebla 72824, México; \\ e-mail:b.posada@inaoep.mx; baldemar@inaoep.mx;ivan.armas@inaoep.mx; ekuz@inaoep.mx \\ ${ }^{d}$ Universidad Autónoma de San Luis Potosí, 78210, México; \\ e-mail:miguel.bello@uaslp.mx \\ e Universidad Autónoma de Nuevo León, San Nicolás de los Garza 66455, México. \\ e-mail: patricia.prietocts@uanl.edu.mx
}

Received 20 November 2020; accepted 2 December 2020

\begin{abstract}
We experimentally demonstrate the generation of mode-locked pulses in a dissipative soliton resonance (DSR) regime. The DSR pulses are obtained from a passively mode-locked figure- $8 \mathrm{Er}$ :Yb doped fiber laser operating in a large net anomalous dispersion regime. The mode-locked laser emission is based on a polarization-imbalanced nonlinear optical loop mirror (NOLM), acting as an artificial saturable absorber. Stable square DSR pulses, obtained by carefully adjusting the NOLM properties, increase their duration from $2.47 \mathrm{~ns}$ to $46.52 \mathrm{~ns}$ as the pump power is increased from 0.68 to $6.31 \mathrm{~W}$. With the maximum pump power launched, an average output power of $73.95 \mathrm{~mW}$ with a pulse energy of $53.39 \mathrm{~nJ}$ is achieved.
\end{abstract}

Keywords: Fiber laser; mode-locked lasers; pulse propagation; dissipative soliton resonance.

PACS: 42.85.Hz; 42.55.Wd; 07.07.Df

\section{Introduction}

In recent years, research on passive mode-locking (PML) techniques for the generation of a wide variety from ultrashort to ultra-wide pulses have been reported [1-10]. Particularly, the study of mode-locked fiber lasers has gained increasing interest because of their intrinsic advantages and attractive properties [2-3]. Techniques such as nonlinear polarization rotation (NPR) [4-6] and different devices such as semiconductor saturable absorber (SESAM) [7], nonlinear optical loop mirror (NOLM) [8], and nonlinear amplifier loop mirror (NALM) [9-10], have been used for passively mode-locked pulse generation in fiber lasers. Different pulse shapes reported in the literature include sech2 pulses [11], self-similar parabolic pulses [12-13], and dissipative soliton resonance (DSR) square pulses [14-15]. In this regard, there is a strong motivation on nonlinear phenomena study in order to obtain specific pulse shapes by designing the cavity configuration and selecting the proper cavity parameters. Different pulses regimes have been demonstrated in mode-locked fiber lasers such as solitons [16,17], dissipative solitons [1113], DSR [4,8,14], solitons rain [15], femtosecond pulses [16, 17], noise-like pulses (NLPs) [18,19], among others. Particularly, generation of square mode-locked laser pulses has been demonstrated in DSR and NLPs operations [5,6,14]. In the case of the laser cavity configurations explored in order to generate and optimize the ultrashort pulsed laser emission, figure-8 laser (F8L) configuration has been demonstrated its versatility and reliability for DSR mode-locked laser pulses generation in fiber lasers. Semaan et al. [20] demonstrated the generation of DSR square pulses in a F8L cavity by coupling two amplifiers with a different pump source. Krzempek et al. [21] reported DSR of an F8L enabling simultaneous amplification of a seed signal to obtain dual-wavelength generation. Furthermore, the pulse energy in mode-locked fiber lasers operating in the anomalous dispersion regime is limited by the area theorem as a function of the pulse shape [22]. However, in the case of DSR operation, a theoretical study based on the numerical solution of the complex cubicquintic Ginsburg-Landau equation predicted that the pulses energy of the DSR pulses could increase indefinitely while its peak power remains constant [23]. Several experimental studies in both anomalous and normal dispersion regime have confirmed the unique properties of DSR pulses, such as long pulse duration, low linear chirp, and emission of high energy pulses [24-26]. DSR pulses have been obtained in different laser cavity configurations with the independence of the mode-locking mechanism [25,27]. Particularly, the use of a nonlinear optical loop mirror (NOLM) as and artificial saturable absorber (SA) offers the advantage of obtaining different pulse shapes by simply modifying the nonlinear transmission characteristics. In this regard, the use of a NOLM 
represents an outstanding advantage, compared with typical SA elements where these characteristics are fixed. Besides the polarization state at the input of the NOLM can be modified; then, the use of a linear polarizer (essential for NPR technique), which may limit the power circulating inside the cavity, is not required. Different approaches are commonly used in order to accumulate different non-linear phase shifts inside the loop, such as the use of an asymmetrical coupler (which leads to different powers of the counter-propagating beams) or the insertion of an optical amplifier asymmetrically placed inside the loop (used in a NALM configuration). In this regard, the use of a NOLM based on polarization imbalance exhibit an attractive advantage compared with the previous methods mentioned above because it offers the possibility to obtain very low transmission from low power components and theoretically to obtain high transmission of high power components by properly adjustment of the birefringent element placed asymmetrically inside the loop. DSR pulses generation from Er:Yb doped fiber offers the possibility of achieving high energy wave-breaking free mode-locked fiber lasers in the region of $1.55 \mu \mathrm{m}$ [10,27-29]. In this paper, we demonstrate the DSR operation of an Er:Yb F8L based on the use of a polarization-imbalanced NOLM used as SA. The experimental results show stable DSR square pulses trains with a repetition rate of $1.385 \mathrm{MHz}$. The pulse width can be tuned from 2.27 to $46.5 \mathrm{~ns}$ by increasing the pump power from 0.68 $\mathrm{W}$ to $6.31 \mathrm{~W}$. For the maximum pump power of $6.31 \mathrm{~W}$, the average output power is $73.95 \mathrm{~mW}$ with a pulse energy of $53.39 \mathrm{~nJ}$.

\section{Experimental setup}

The experimental setup of the passivly mode-locked fiber laser is shown in Fig. 1. The mode-locking mechanism of the F8L cavity is based on the use of a NOLM as SA. The NOLM is formed by a 50/50 coupler whose output ports are interconnected by $120 \mathrm{~m}$ of SMF-28 fiber twisted at a rate of
7 turns per meter and a quarter-waver retarder (QWR). Fiber twist eliminates the fiber residual birefringence, preserving the ellipticity during the light propagation. Since a symmetric coupler is used, polarization imbalance in the NOLM is induced by using a quarter-wave retarder (QWR2). For this purpose, QWR2 has placed near a 50/50 coupler to breakup the symmetry of the loop and achieve different nonlinear phase shifts of counter-propagating pulses [30]. Because of the power symmetry of the coupler, low power transmission can be adjusted by rotation of QWR2 to reach a very low level. Therefore, the NOLM allows the possibility of change low power transmission and also nonlinear dependence of the transmission just by rotation of QWR2.

The cavity includes a $1.8 \mathrm{~m}$ long Er:Yb double-clad fiber (EYDCF, CorActive DCF-EY-10/128) with core numerical aperture (NA) of 0.20 , a diameter of $10 \mu \mathrm{m}$, and absorption of $85 \mathrm{~dB} / \mathrm{m}$ at $1535 \mathrm{~nm}$, and inner clad diameter of $128 \mu \mathrm{m}$, absorption of $2 \mathrm{~dB} / \mathrm{m}$ at $915 \mathrm{~nm}$ and $\mathrm{NA}>0.45$. The laser cavity also includes a polarization-dependent isolator (PDISO) to ensure unidirectional light propagation and providing stable linear polarization at a quarter-wave-retarder (QWR1). Without PD-ISO, the polarization may be altered by the EYDCF birefringence, which depends on the pump power because of the thermal effect [30]. The QWR1 is placed after the PD-ISO to change the light polarization at the input of the NOLM.

Different input polarizations lead to different NOLM operation characteristics. In the experiment, the angle of the QWR1 was placed at $45^{\circ}$ for the axis of the PD-ISO to provide circular polarization of light at the NOLM input. The advantages of using circular polarization at the input of the NOLM were described by Barmenkov et al. in Ref. [31]. The $10 \%$ output port of a 90/10 optical fiber coupler placed at the NOLM output was used to provide the laser output. A $90 \%$ port connected to the input signal port of the beam combiner closes the laser cavity. The EYDCF is

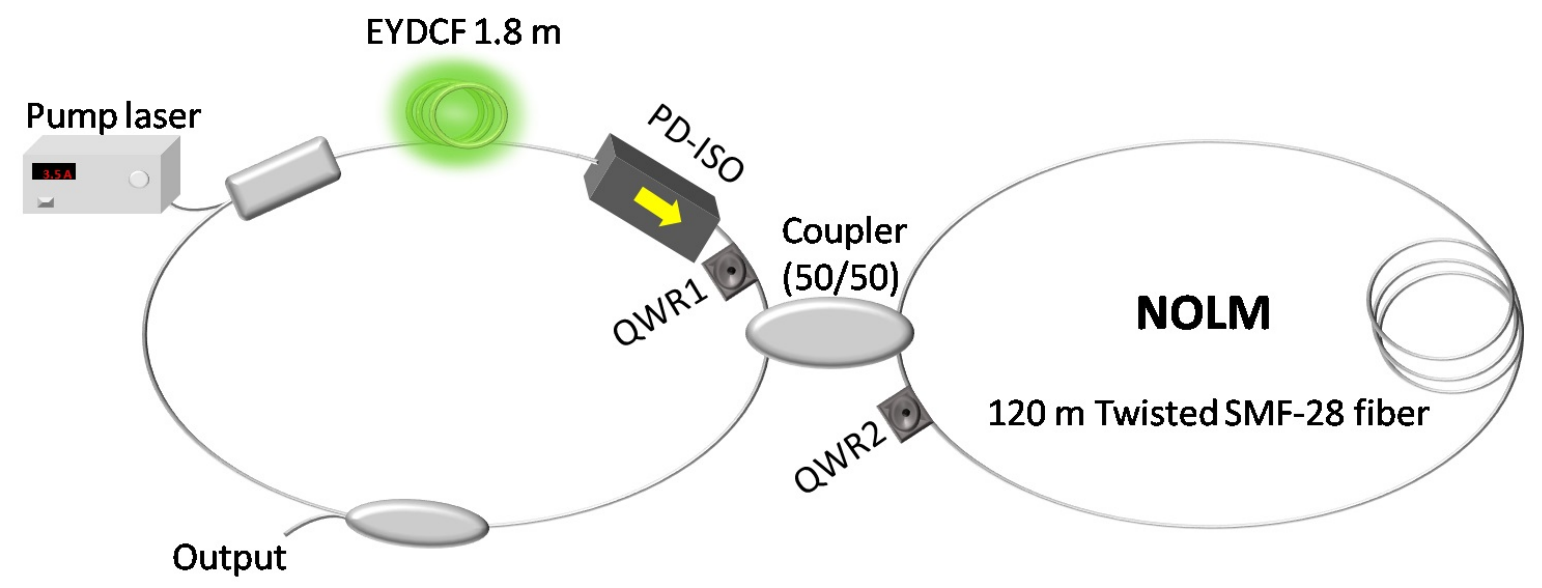

FIGURE 1. Experimental setup of the EYDCF mode-locked F8L, based on the use of a NOLM as SA. 


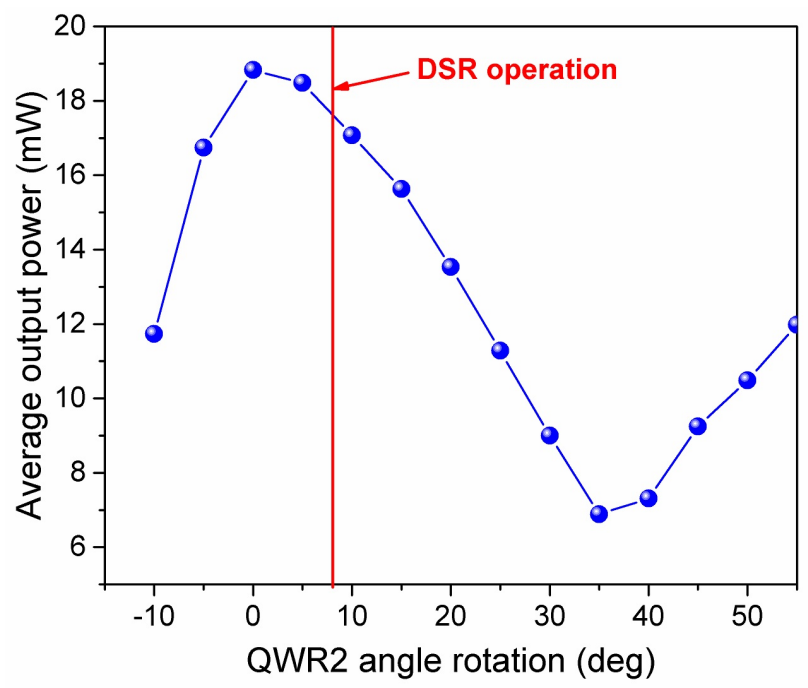

FIGURE 2. Average output power as a function of the QWR2.

pumped by a $25 \mathrm{~W}$ multimode laser source at $976 \mathrm{~nm}$ through a $(2+1) \times 1$ beam combiner. The maximal launched pump power was $6.31 \mathrm{~W}$. The laser output power is measured by a thermal optical power meter (Thorlabs PM310D). The output pulses are detected by a high-speed photodiode (12.5 $\mathrm{GHz}$ bandwidth and $28 \mathrm{ps}$ rise/fall time) and monitored by a real-time $2.5 \mathrm{GHz}$ bandwidth oscilloscope. The optical output spectrum was measured by an optical spectrum analyzer (OSA, Yokogawa AQ6375) with a scanning range from 1200 to $2400 \mathrm{~nm}$ and maximal resolution of $0.05 \mathrm{~nm}$. The radiofrequency (RF) spectrum of the output pulses is detected by a high-speed photodiode and measured by using a spectrum analyzer (Agilent, N9344C) with a resolution bandwidth from $10 \mathrm{~Hz}$ to $3 \mathrm{MHz}$ and frequency span range from $100 \mathrm{~Hz}$ to 20 $\mathrm{GHz}$. The estimated total cavity length is $\sim 148$, and the estimated net cavity dispersion is $-3.10 \mathrm{ps}^{2}$. Then, the proposed laser is operating in an anomalous dispersion regime.

\section{Results}

Self-started mode-locking operation of the F8L is reached when the pump power is increased to $0.68 \mathrm{~W}$. Then, with the proper adjustments of QWR1 and QWR2, a stable DSR mode-locking operation is obtained. In order to characterize the QWR2 conditions in which DSR is achieved, the average output power as a function of the QWR2 angle was measured, as shown in Fig. 2. The pump power was set at $6.31 \mathrm{~W}$. As it can be observed, the rotation of QWR2 modifies the output power of the laser due to the transmission variation in the NOLM. The zero-degree angle corresponds to the maximum low power of the NOLM transmission. DSR pulses were obtained when the QWR2 plate was fixed at an eight degrees angle.

Figure 3 shows the characteristics of DSR mode-locked laser emission at the maximum pump power of $6.31 \mathrm{~W}$. In Fig. 3a), fundamental mode-locking is confirmed by the appearance of a stable train of pulses with a period of $721 \mathrm{~ns}$, i.e., a single pulse circulates in the $148 \mathrm{~m}$ long cavity. The measurement of a single pulse in Fig. 3b) exhibits a square waveform of the pulse profile with a duration of $46.52 \mathrm{~ns}$. The slightly asymmetrical amplitude is a typical characteristic of some DSR mode-locked lasers [10,24,30]. The optical spectrum of the DSR mode-locked laser emission is shown in Fig. 3c). The optical spectrum shows a smooth shape profile with central wavelength at $1564 \mathrm{~nm}$ and full width at half maximum (FWHM) of $4.8 \mathrm{~nm}$. The measured optical spectrum is well fitted to a Gaussian function.

Figure 4 shows the characteristics of the DSR laser generation as a function of the pump power in the range from 0.68 to $6.31 \mathrm{~W}$. Figure 4a) shows the optical spectra centered at $1564 \mathrm{~nm}$. As it can be observed, the profile of the optical spectra and the FWHM bandwidth are the same over all the pump power range. As it can be expected from DSR laser operation, the duration of the squared pulses increases from 2.47 to $46.52 \mathrm{~ns}$ as the pump power increases from 0.68 to $6.31 \mathrm{~W}$, as shown in Fig. 4(b). As it was theoretically predicted in Ref. [31] and experimentally confirmed in Ref. [32], the obtained DSR pulses exhibit low linear chirp across the pulse, except to the observed at the edges, which shows exponentially variation of chirp.

Since the autocorrelation trace of the square pulse aims towards constant level due to the low scanning range (200 ps) compared to the pulse duration $(\sim n s)$, in order to confirm stable DSR laser operation, the RF spectrum was measured
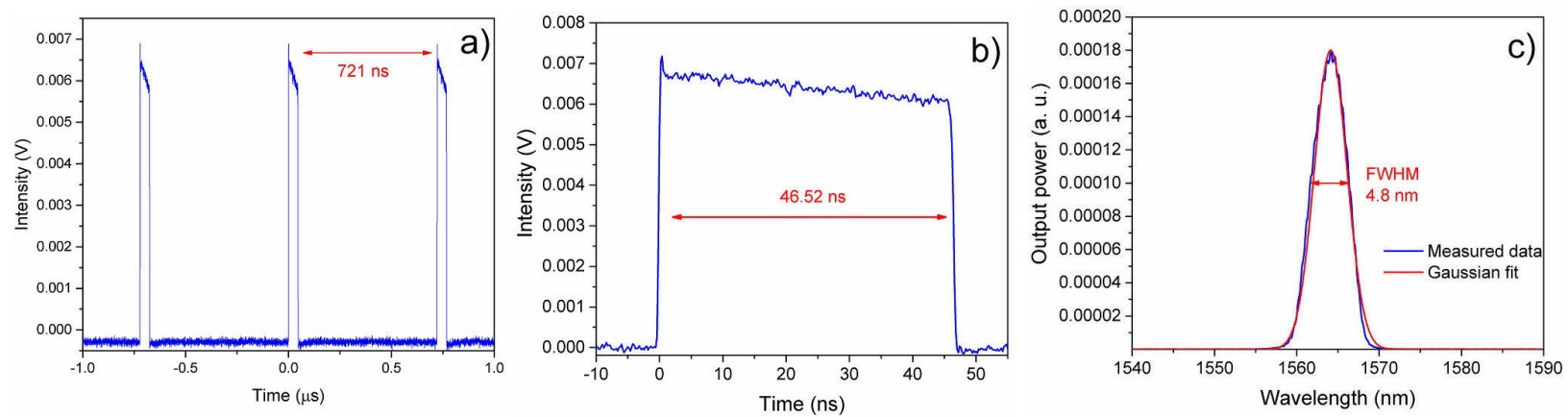

FIGURE 3. Square-wave pulse emission of the laser for maximum pump power of $6.31 \mathrm{~W}$ : a) Pulses train, b) temporal profile of a single DSR pulse, c) optical spectrum of the DSR pulse. 

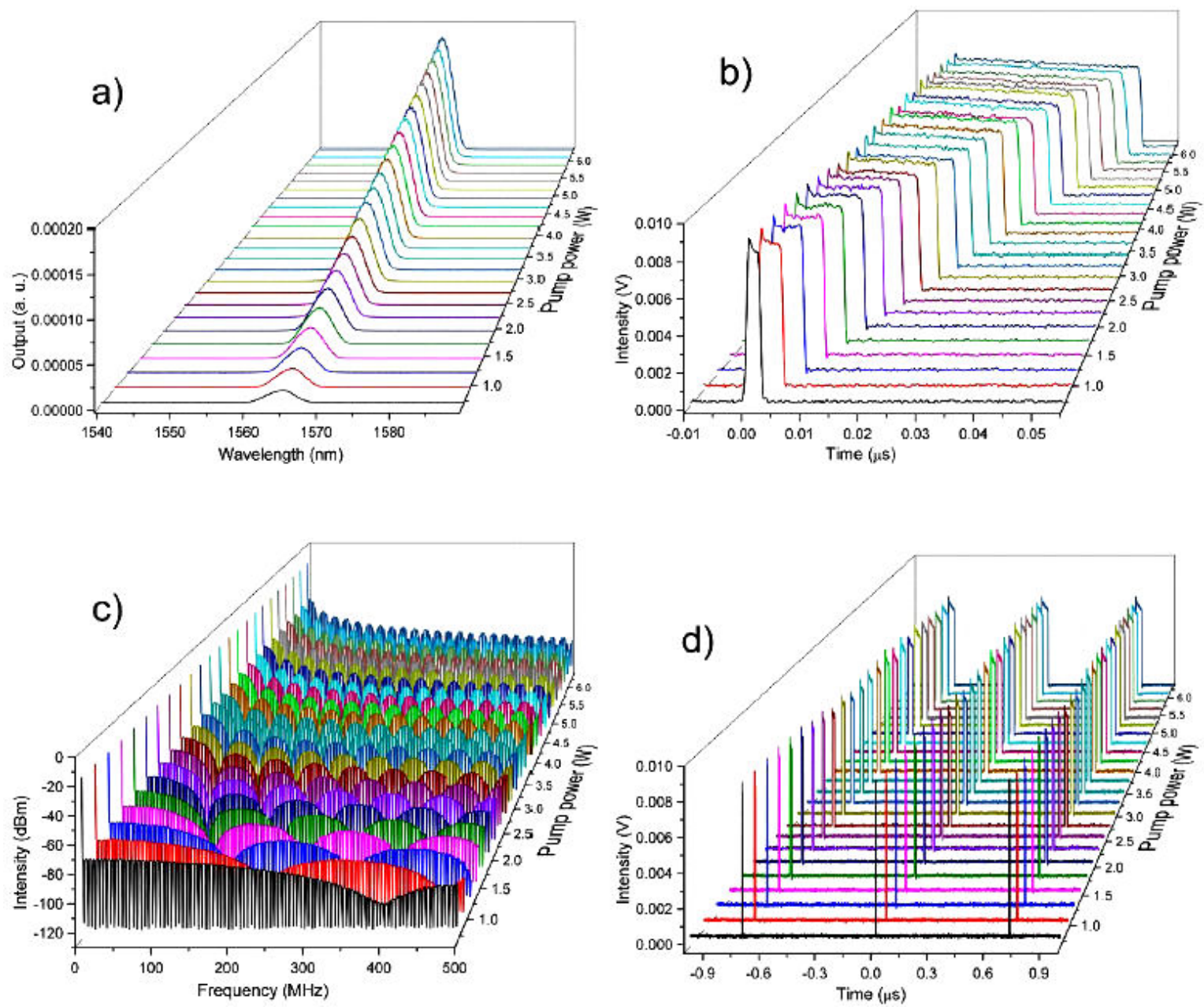

FIGURE 4. DSR laser characteristics on pump power variations: a) optical spectra, b) evolution of rectangular pulses. c) RF spectra, d) trains of pulses.

with a $100 \mathrm{MHz}$ span and $1 \mathrm{kHz}$ resolution. As it can be observed in Fig. 4(c), when the pump power is increased from 0.68 to $6.31 \mathrm{~W}$, the frequency of the first power minimum decreases from $400 \mathrm{MHz}$ to $21.4 \mathrm{MHz}$, related to pulse duration variations from $\sim 2.5 \mathrm{~ns}$ to $\sim 46.7 \mathrm{~ns}$. Thus, the modulation pattern is defined by the duration of the generated pulses, which corresponds to the reciprocal value of the pulse duration. Figure 4d) shows stable trains of pulses in a pump power range from 0.68 to $6.31 \mathrm{~W}$, with a fundamental repetition rate of $1.386 \mathrm{MHz}$.

Figure 5 shows the parameters of the generated DSR pulses as a function of the pump power. Figure 5a) shows an increasing linear dependence of the average power and the pulse energy with the pump power increase. At the maximum pump power of $6.31 \mathrm{~W}$, the measured average output power is of $73.95 \mathrm{~mW}$ and the estimated pulse energy is $\sim 53.39 \mathrm{~nJ}$. As it can be observed in Fig. 5b), the duration of the square pulses increases from 2.47 to $46.5 \mathrm{~ns}$ as the pump power is increased. Then, considering a pulse repetition rate of 1.385 $\mathrm{MHz}$, the calculated peak power of $\sim 1.15 \mathrm{~W}$ remains constant independently of the pump power level, which is a dis- tinctive operation characteristic of DSR laser emission.

\section{Conclusions}

In summary, we demonstrated DSR effects in a passively mode-locked figure-eight Er:Yb doped fiber laser. With proper settings of the QWR1 and QWR2, self-started modelocking is reached at the pump power level of $0.68 \mathrm{~W}$. With the increase of the pump power from $0.68 \mathrm{~W}$ to $6.31 \mathrm{~W}$, DSR square pulses with a repetition rate of $1.385 \mathrm{MHz}$, pulse width in a range from $2.47 \mathrm{~ns}$ to $46.5 \mathrm{~ns}$, average power from $3.35 \mathrm{~mW}$ to $73.95 \mathrm{~mW}$, and pulse energy from 2.41 to 53.39 $\mathrm{nJ}$, are obtained.

\section{Acknowledgements}

M.D.-S wants to thank Cátedras CONACyT. R.I.A.-T was supported in part by Fondo de Investigación UPAEP 2020 project. P.P.-C. wants to thank the CONACyT postgraduate scholarship, grant no. 160540 . 
1. A. B. Grudinin and S. Gray, J. Opt. Soc. Am. B, 14 (1997) 144. https://doi.org/10.1364/JOSAB.14.000144

2. W. Shi, Q. Fang,X. Zhu, R. A. Norwood and N. Peyghambarian, Appl. Opt, $\mathbf{5 3}$ (2014) 6554.https://doi.org/10. $1364 / \mathrm{AO} .53 .006554$

3. U. Keller, Nature, 424 (2003) 831. https://doi.org/ $10.1038 /$ nature01938

4. V. J. Matsas, T. P. Newson, D. J. Richardson, and D. N. Payne, Electron. Lett., 28 (1992) 1391. https://doi.org/10. $1364 / \mathrm{OL} .18 .000358$

5. H. Santiago-Hernández et al., Opt. Express, 25 (2017) 25036. https://doi.org/10.1364/OE.25.025036

6. L. A. Rodríguez-Morales et al., Opt. Express, 27 (2019) 14030. https://doi.org/10.1364/OE.27.014030

7. L. Yun, X. M. Liu, and D. Mao, IEEE J. Sel. Top. Quant., 20 (2012) 20992

8. K. Krzempek, and K. Abramski Laser Phys. Lett., 14 (2017) 015101. https://doi.org/10.1088/1612-202X/ $14 / 1 / 015101$

9. W. Shi-Ke, N. Qiu-Yi, L. Ai-Ping, L. Zhen-Bin, L. Zhi-Chao, and X. Wen-Cheng, Opt. Express, 21 (2013) 2402. https: //doi.org/10.1364/OE.21.002402

10. K. Krzempek, and K. Abramski, Opt. Express, 24 (2016) 22379. https://doi.org/10.1364/OE.24.022379

11. H. A. Haus, IEEE J. Sel. Top. Quantum Electron., 6 (2000) 1173 .

12. A. Chong, J. Buckley, W. Renninger, and F. Wise, Opt. Express, 14 (2006) 10095. https://doi.org/10.1364/ OE.14.010095

13. F. Ö. IIday, J. R. Buckley, W. G. Clark, and F. W. Wise, Phys. Rev. Lett., 92 (2004) 213902. https://doi.org/ 10.1103/PhysRevLett.92.213902

14. L. Duan, X. Liu, D. Mao, L. Wang, and G. Wang, Opt. Express., 20 (2012) 265. https: / /doi.org/10.1364/OE. 20.000265

15. N. Akhmediev, J. M. Soto-Crespo, and P. Grelu, Phys. Lett. A., 372 (2008) 3124. https://doi.org/10.1016/j. physleta.2008.01.027

16. A. B. Grudinin, D. J. Richardson, and D. N. Payne, Electron. Lett., 29 (1993) 1860.

17. F. Amrani, M. Salhi, P. Grelu, H. Leblond, and F. Sanchez, Opt. Lett, 36 (2011) 1545. https : //doi.org/10.1364/ OL.36.001545
18. O. Pottiez et al., Laser Phys, 24 (2014) 115103. https: //doi.org/10.1088/1054-660X/24/11/115103

19. H. Santiago Hernández et al., Opt. Express, 23 (2015) 18840. https://doi.org/10.1364/OE.23.018840

20. G. Semaan, F. B. Braham, J. Fourmont, M. Salhi, F. Bahloul, and F. Sanchez, Opt. Lett., 41 (2016) 4767. https://doi. org/10.1364/OL.41.004767

21. K. Krzempek, G. Sobon, J. Sotor, and K. M. Abramski, Opt. Express, 23 (2015) 32080. https: / / doi .org/10.1364/ OE.23.032080

22. W. H. Renninger, A. Chong, and F. W. Wise, J. Opt. Soc. Am. B, 27 (2010) 1978. https://doi.org/10.1364/JOSAB. 27.001978

23. W. Chang, A. Ankiewicz, J. M. Soto-Crespo, and N. Akhmediev Phys. Rev. A., 78 (2008) 023830. https: / /doi.org/ $10.1103 /$ PhysRevA. 78.023830

24. I. Armas-Rivera et al., Opt. Express, 24 (2016) 9966. https : //doi.org/10.1364/OE.24.009966

25. K. Krzempek, D. Tomaszewska, and K. M. Abramski, Opt. Express, 25 (2017) 24853. https: / / doi.org/10.1364/ OE.25.024853

26. K. Krzempek, J. Sotor, and K. Abramski, Opt. Lett., 41 (2016) 4995.https://doi.org/10.1364/OL.41.004995

27. G. Semaan, F. B. Braham, M. Salhi, Y. Meng, F. Bahloul, and F. Sanchez, Opt. Express, 24 (2016) 8399. https://doi. org/10.1364/OL.41.004767

28. K. Krzempek, Opt. Express, 23 (2015) 30651. https:// doi.org/10.1364/OE.23.030651

29. G. Semaan, A. Niang, M. Salhi, and F. Sanchez, Laser Phys. Lett., 14 (2017) 055401. https://doi.org/10.1088/ $1612-202 \mathrm{x} / \mathrm{aa} 6700$

30. O. Pottiez, E. A. Kuzin, B. Ibarra-Escamilla, and F. MéndezMartínez, Opt. Commun, 229 (2004) 147. https://doi. org/10.1016/j.optcom.2005.05.015

31. Y. O. Barmenkov, A. V. Kir'yanov, and M. V. Andrés Appl. Phys. Lett., 85 (2004) 2466. https : //doi.org/10. $1063 / 1.1787151$

32. X. Li, X. Liu, X. Hu, L. Wang, H. Lu, Y. Wang, and W. Zhao, Opt. Lett., 35 (2010) 3249. https : / / doi.org/10.1364/ OL.35.003249 